\title{
Intervención al duelo por ruptura de pareja desde la terapia cognitivo conductual a partir del análisis de cuatro casos
}

\author{
Intervention in grief at the rupture of a couple relationship \\ using conductive cognitive therapy, based on four case analyses
}

\section{Resumen}

La investigación buscó identificar las principales respuestas de duelo frente a la pérdida que implica la ruptura de pareja y las técnicas de intervención a partir de la revisión de cuatro historias clínicas. Las respuestas comunes fueron: diálogos agonizantes sobre el rol cumplido por el otro, sobre el proyecto de vida y daños recibidos; culpa, transformación del autoconcepto, de la noción de amor y pareja. Atribuir la responsabilidad de la perdida a acciones realizadas por el otro. Cuando la separación es motivada por infidelidad, se mantiene la negación de la pérdida. La técnicas principales fueron: empatía, clarificar el rol cumplido de la ex pareja, descongestión emocional, guías deliberativas sobre vida, amor, divorcio, matrimonio, dignidad; reestructuración sobre atribuciones disfuncionales de la pérdida, revisión del rol actual hacia la pareja y fortalecimiento del amor propio.

Palabras clave: duelo, pareja, análisis de caso.

\begin{abstract}
The study sought to identify the main responses to grief at the loss deriving from the rupture of a relationship, and the intervention techniques used to treat it, based on the review of four clinical histories. The common responses were agonizing dialogs on the role played by the other in the patient's life project, and the harm received; guilt, transformation of the self-concept and the notion of love and partner. Responsibility for the loss is attributed to actions by the other party. When the separation is caused by infidelity the negation of loss is maintained. The principal techniques applied were empathy, clarifying of the role played by the ex-partner, emotional decongestion, deliberative guides on life, love, divorce, marriage, and dignity; re-structuring dysfunctional attributes of the loss, reviewing the role the partner had played and strengthening of self-esteem.
\end{abstract}

Keywords: grief, partner, case analysis.

Recibido el 13 de mayo de 2012 y aprobado el 12 de julio de 2012

1 Cristina Isabel Carmona es psicóloga egresada de la Universidad Nacional de Colombia. Magister en Psicología Clínica con Énfasis Cognitivo Conductual de la Universidad Católica de Colombia. 


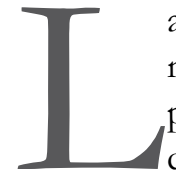

a terapia cognitiva-conductual propone modelos de intervención a corto plazo, activos, directivos, estructurados, colaboradores y psicoeducacionales, centrados en ayudar al paciente a examinar la manera en la que construye y entiende el mundo (cogniciones), así como experimentar con nuevas formas de respuesta (conductual). Su principal objetivo consiste en transformar percepciones negativas, desviadas o distorsionadas de la persona acerca de sí misma, los demás y el mundo, de modo que pueda pensar y actuar de un modo más realista y adaptativo, en relación con sus problemas psicológicos, reduciendo o eliminando, así, los síntomas hasta alcanzar sus metas. Este proceso proporciona al paciente una mayor sensación de predicción y control. (Freeman \& Reinecke, 1995, citado por Riso, 2006). Quiere decir que la psicoterapia tiene la posibilidad de transformar pensamientos, afectos y comportamientos (Cozolino, 2003).

El estudio tuvo como objetivo identificar técnicas de intervención psicoterapéutica al duelo en adultos a partir de la caracterización de respuestas comunes a nivel cognitivo, afectivo y motor de dolientes adultos. Se utilizó una metodología de análisis de caso en cuatro pacientes, que asistieron a consulta por ruptura de pareja a dos instituciones de atención clínica en la ciudad de Bogotá. Como criterio de inclusión se tomó la elección sexual, debido a que hay autores que evidencian particularidades en las manifestaciones afectivas cuando el participante tiene una elección homosexual. Así lo confirman Gotta, Green,
Rohblum, Solomon, Balsam y Schaertz (2011), al encontrar diferencias significativas en parejas constituidas por hombres gay, mujeres lesbianas y personas heterosexuales en tareas compartidas dentro del hogar, toma de decisiones, formas de apoyo, comunicación y actitud frente al sexo. De este modo, si las características afectivas en una relación entre homosexuales tienen matices diferenciales respecto a una pareja homosexual, el duelo resultante de la ruptura tendrá también diferencias. Para algunos autores, estas diferencias obedecen más a prejuicios sociales. No obstante, la discusión está abierta.

La pertinencia de estudios guiados a identificar cogniciones y comportamientos está en el hecho de que estas respuestas son mantenedoras de síntomas que pueden llevar a la complicación o recuperación de la salud mental de los pacientes. Así lo comprueban Boelen, de Keijser, Vanden Hout y Van den Bout (2011), al verificar que 43 pacientes con duelo patológico mejoraban significativamente en la medida en que iban siendo reestructuradas sus creencias irracionales y modificadas sus conductas maladaptativas. Así mismo, en el 2006, Boelen, Van den Bout y Van den Hout trabajaron la asociación entre variables cognitivas y la complicación emocional en el transcurso de elaboración de la pérdida. De otro lado, Boelen y Van den Bout (2002) encontraron en 326 adultos cómo el pensamiento positivo era inversamente proporcional a la aparición de depresión y duelo patológico, demostrando, así, el papel influyente de los pensamientos y conductas en el proceso de elaboración de la perdida. 
En síntesis, se ha comprobado la efectividad de este tipo de terapia en casos de duelo (Cohen, Mannarino, \& Knudsen, 2004).

Adicional a lo anterior, autores como Love (2007) mencionan la alteración que las perdidas generan a nivel de creencias sobre uno mismo, el mundo y lo que va a ocurrir a futuro.

La conceptualización tomada en cuenta para la investigación obedece al modelo de formulación cognitivo conductual del Dr. Walter Riso (2006), promovido como Análisis Multinivel. Según el autor, la psicopatología del paciente se estudia a partir de tres estructuras de razonamiento. Una, sintomática/descriptiva. La segunda, explicativa/preventiva y la tercera, explicativa/promocional. Los tres actúan de manera integrada, pero se dividen por el carácter explicativo necesario para la intervención. El primer nivel muestra el papel del medio ambiente en la elicitación de respuesta a nivel cognitivo, afectivo y motor, así como las consecuencias obtenidas a partir de éstas. El análisis inicial consiste en la descripción detallada de los estímulos que participan en la situación problemática del paciente. Luego se identifican los pensamientos automáticos y distorsiones cognitivas evidenciadas, señalando las emociones y acciones correlacionadas con éstos. Finalmente, se analiza la funcionalidad de las respuestas en términos contingentes, es decir, son reforzantes o punitivas (castigo), que, según los principios de la terapia conductual, dan cuenta del mantenimiento de la conducta. Esta primera estructura responde al cómo sucede el problema reportado por el paciente. Por ejemplo, un paciente que ha tenido una ruptura de pareja significativa menciona que se ha sentido triste, sin ganas de empezar el día y se distrae fácilmente en medio de su trabajo. Al indagar, se observa que la vinculación laboral que refiere no le satisface lo suficiente debido a problemas interpersonales. (Grupo de Investigadores en psicología UMB, 2008).

En la situación anterior se identifica en el primer nivel de análisis:

Estimulo discriminativo: Hora de la mañana.

\section{Respuestas:}

Cognitiva: "No tengo la suficiente fuerza para continuar". "No tengo lo que se necesita para lograr estar bien".

Afectiva: Tristeza.

Comportamental: Baja la velocidad, el ritmo en sus labores, disminuye el contacto con sus compañeros de trabajo.

Consecuencia: Refuerzo negativo, evita confrontar un ambiente laboral que no le satisface.

El segundo nivel de análisis permite explicar porqué se presentan las estructuras contingentes descubiertas en el primero, que desde el enfoque cognitivo-conductual se asocian a un sistema de procesamiento de información inadecuado. En este segundo nivel se analizan las creencias centrales o esquemas nucleares que pueden entenderse como el conjunto de teorías con las que el paciente explica el mundo. Según el autor, los esquemas buscan ser perpetuados por las creencias intermedias. Es decir, la persona conserva unas reglas rígidas que se expresan en términos de deberías y silogismos que le sirven para evaluar las situaciones que enfrenta, asegurando que la interpretación dada por el sujeto coincida con la información preexistente contenida en el esquema. En el ejemplo anterior podría identificarse una regla del tipo:

Regla: Debido a que hay algo insuficiente, inadecuado e incapaz en mí, los demás terminarán abandonándome.

Las reglas a su vez se protegen por estrategias compensatorias del tipo profecía auto-cumplida o estrategia evitativa. Estas son comportamientos que la persona realiza con el ánimo de no enfrentar la información que contradiga la dictada por sus esquemas y reglas. Por ejemplo, la acción de no comunicar sus sentimientos de duelo con los que posiblemente obtendría reconocimiento y consideración de los demás.

Finalmente, el tercer nivel considerado como una estructura superior. Llamada esquemas de segundo orden. Contempla la necesidad de eva- 
luar el sistema de creencias asociado a la dimensión trascendente del ser humano y se evalúa especialmente toda vez que el paciente reporta o se evidencia sintomatología relacionada con cuestionamientos de tipo existencial.

La formulación del segundo y tercer nivel no serán tomados en cuenta por el carácter particular que tiene en cada uno de los casos.

\section{Metodología}

El presente estudio hace parte de una investigación macro sobre protocolos de intervención al duelo en adultos, realizada con el grupo de Muerte y Duelo de la Universidad Manuela Beltrán.

La técnica de análisis de caso consiste en una forma de recolección de información a partir de la selección de un ejemplar representativo al fenómeno de estudio. Posteriormente, se realiza el análisis cualitativo, estableciendo las categorías con las cuales será comprendido el fenómeno. La presente investigación utiliza el registro de archivos, específicamente historias clínicas (Martínez, 2006).

Las categorías que se tuvieron en cuenta son:

Respuestas cognitivas: Consiste en los pensamientos automáticos señalados por el paciente al enfrentar los estímulos discriminativos.

Respuestas afectivas: Se refiere a los sentimientos y emociones señalados por el paciente al enfrentar los estímulos discriminativos.

Respuestas comportamentales: Se refiere a las acciones realizadas por el paciente al enfrentar los estímulos discriminativos.

Técnicas de intervención: Se refiere a las acciones terapéuticas.

Se escogieron cuatro participantes que son llamados de manera indiferenciada, paciente o doliente que hace referencia a la persona que ha tenido una pérdida significativa, por lo cual está en proceso de duelo y asiste a consulta.

\section{Participante 1}

Hombre de 49 años, profesional especialista.

Tipo de pérdida: Separación afectiva con su esposa luego de 21 años de matrimonio, mediante un proceso anunciado a través de varios intentos por salvar la relación.

Religión: Católica. Practica ejercicios constantes de fortalecimiento espiritual.

\section{Resumen de la Historia.}

El doliente refiere relación de pareja por vínculo matrimonial de 20 años, caracterizada por la excesiva dedicación al trabajo como expresión de amor para con su familia. La preocupación por una estabilidad económica apropiada lo mantuvo alejado de las problemáticas cotidianas y deseos de su núcleo. Esta situación se complejiza por un ejercicio de la fe católica, exigente a nivel de autocontrol, que implican de parte del paciente conductas de indiferencia y abandono hacia su esposa. La práctica religiosa del doliente transformó su visión de situaciones antes valoradas como proveedoras de placer, pero que fueron adquiriendo el calificativo de pecaminosas, cambiando así el valor del estímulo consecuente, de reforzadores pasaron a ser aversivos. De este modo, las situaciones sociales compartidas por la pareja fueron diezmándose al ser descalificadas por el paciente. La pareja reporta problemas sexuales de inicio temprano, asociados a la baja satisfacción de su esposa, así como dificultades en las relaciones con familia extensa, especialmente con la figura materna del doliente, a quien le resulta incómodo las manifestaciones afectivas hacia su nuera y nietos. La relación empieza a deteriorarse 10 años antes de la ruptura, con el evento desencadenante de una cirugía estética que puso en riesgo la vida de su esposa. A partir de ahí, la pareja inicia intentos de negociación frente a temas relacionados con estilo de vida, debido a que no comparten la visión de la fe del paciente. La esposa del doliente evita el conflicto y durante años conserva en silencio sus inconformidades, explicando su conducta a partir de la resistencia de su esposo al cambio. Actualmente, ella se muestra segura de su decisión de separar- 
se, no obstante, experimenta altos niveles de culpabilidad que resultan reforzados por el discurso religioso de su esposo, sobre la legitimidad del matrimonio. Él intenta reconquistarla con detalles que reconoce haber perdido, pero se muestra resistente a negociar sus prácticas religiosas.

\section{Tabla No. 1. Intervención participante 1. No. de sesiones: 12}

\begin{abstract}
Actividad realizada
1. Se utilizaron preguntas del tipo: (a) ¿Podrías contarme que sucedió la última vez que hablaste con tu pareja o relatarme los sucesos con la mayor exactitud que te sea posible? (b) ¿Cuáles eran los reclamos de tu pareja y hasta qué punto atendiste a ellos? Cuéntame con exactitud cómo era tu relación de pareja.
\end{abstract}

2. Historización de la relación desde el noviazgo, para lo cual se solicita al paciente llevar al consultorio fotos y música que le permitan recordar lo vivido.

3. Describir con detalle su rutina de vida, haciendo énfasis en los momentos en que aparece su pareja. Se le pide al paciente especificar con detalle los aspectos asociados a la falta del otro, es decir, los tiempos, espacios, experiencias que se extrañan.

4. Como el motivo de consulta tiene que ver con ambivalencia, se le dejan tareas terapéuticas asociadas al balance decisional.

5. A lo largo de la terapia se trabajó con esquemas de segundo orden, específicamente asociados a la religiosidad del paciente por la influencia de estas en su forma de relacionarse y su resistencia a la separación.

6. Análisis sobre actitudes personales que intervinieron en la separación.

7. Se le pide realizar una carta a su ex pareja, señalando las heridas causadas y realizar un acto desde su fe que pueda ayudar en la reparación de esas heridas.

8. Hacia la VIII y IX sesión se dirige la mirada del paciente a sí mismo. De este modo se le invita a fortalecer su autoimagen y a pensar en su proyecto de vida.

9. Se rescata el valor de la relación terapéutica.

\section{Objetivo terapéutico}

Reestructurar las atribuciones disfuncionales ante la responsabilidad de la pérdida

Facilitar la expresión emocional y organizar el discurso alrededor de la pérdida. Autores como Crenshaw (2006) refieren que un discurso organizado alrededor de experiencias difíciles y traumáticas suelen ayudar en el proceso de elaboración.

Psicoeducación en la identificación de estímulos discriminativos elicitadores de malestar psicólogo y facilitar la expresión emocional.

Facilitar el proceso de toma de decisiones asociadas a la reconstrucción de un proyecto de vida sin pareja.

Concienciar al paciente de la disfuncionalidad de algunos ejercicios de fe, para promover habilidades sociales con las cuales pueda compartir más efectivamente su experiencia espiritual.

Promocionar un repertorio de conductas más funcional para el mantenimiento de relaciones de pareja futuras.

Minimizar el filtraje que existe frente a los aspectos positivos de la relación para facilitar la elaboración de la pérdida.

Minimizar la visión de túnel frente a la posibilidad de volver a experimentar felicidad y aumentar el nivel de bienestar actual.

Facilitar la expresión emocional, minimizar la culpa y reestructurar la visión negativa que se tiene sobre sí mismo. 


\section{Participante 2}

Mujer de 39 años. Trabaja como secretaria.

Tipo de pérdida: Ambigua. Separación afectiva con su esposo luego de 18 años de matrimonio. El evento fue progresivo y actualmente mantienen una relación de "amistad", según lo refiere la paciente. Ella cumple el papel de consejera respecto a la nueva relación establecida por su ex esposo.

\section{Religión: Católica.}

\section{Resumen de la Historia.}

La paciente tuvo una relación de 18 años, caracterizada por ausencia de conflictos, pero extrema complacencia de su parte, tanto que los roles resultaron desvirtuados y ella empieza a comportarse más como una madre que como esposa. Los límites hacia las exigencias de su esposo eran pocos, incluso dejó de pedir caricias y los intercambios a nivel afectivo, sexual, económico y comunicacional resultaban desequilibrados. Por otro lado, la doliente declara haberse dedicado excesivamente a su trabajo, donde ocupa un lugar de prestigio por su buen desempeño. Desde hace tres años ella descubre infidelidad en su esposo, reaccionando con cambio de rol, pasando de ser esposa/madre a consejera, y permitiendo encuentros ocasionales con su amante, hasta que él decide mudarse de casa argumentando que se va a organizar con su nueva compañera. Actualmente tienen encuentros sexuales ocasionales y mantienen el rol confidente-confesor.

A propósito de la expresión emocional facilitada en la historización y la identificación de estilos de afrontamiento a partir de la revisión de rutinas, autores como Heiney, Dunaway \& Webster (1995) trabajaron con niños con duelo por muerte, evidenciando que estas dos técnicas ayudaban en la remisión de síntomas.

Tabla No. 2. Intervención participante 2. No. de sesiones: 1, duración de 3 horas

\begin{tabular}{|l|l|}
\hline \multicolumn{1}{|c|}{ Actividad realizada } & \multicolumn{1}{c|}{ Objetivo terapéutico } \\
\hline $\begin{array}{l}\text { Historizar. El valor de la expresión emocional de senti- } \\
\text { mientos asociados al duelo es soportada por autores } \\
\text { como Kranzler, Shaffer, Wasserman \& Davies (1990). }\end{array}$ & $\begin{array}{l}\text { Sensibilizar a la paciente frente al daño que la relación } \\
\text { le había causado y que ella no está viendo por el sesgo } \\
\text { derivado del filtro cognitivo. Etiquetación: "Soy mujer de un } \\
\text { solo hombre". Del mismo modo, se busca que la paciente } \\
\text { comprendiera que su ex esposo había tomado una decisión. }\end{array}$ \\
\hline Revisión de estilo de vida actual. & $\begin{array}{l}\text { Mostrar a la paciente que su estilo de vida actual carecía } \\
\text { de reforzadores suficientes y por tal motivo ver a su esposo, } \\
\text { se convertía en su único reforzador. Un segundo objetivo } \\
\text { consistía en motivarla a transformar su rutina diaria en la } \\
\text { medida en que evidencia un patrón de afrontamiento a la } \\
\text { perdida disfuncional. }\end{array}$ \\
\hline Revisión del rol actual con su pareja. & $\begin{array}{l}\text { Evidenciar la disfuncionalidad del vínculo actual para } \\
\text { guiar toma de decisiones mucho más acorde con su meta: } \\
\text { "Dejarlo ir". Del mismo modo, se buscaba que la paciente } \\
\text { descubriera el dolor que generaba este tipo de vínculo. }\end{array}$ \\
\hline $\begin{array}{l}\text { Guías deliberativas sobre el amor. } \\
\text { Reestructuración cognitiva de la idea irracional: "Soy mujer } \\
\text { de un solo hombre". }\end{array}$ \\
\hline
\end{tabular}




\section{Participante 3}

Mujer de 38 años, trabajadora técnica.

Tipo de ruptura: Paulatina luego de unión libre con hijos. Se conocieron desde que estaban en segundo de primaria.

Resumen de historia.

En la paciente se evidencian factores predisponentes de historia de exclusión por parte de su círculo social, lo que marca un deterioro en su auto-concepto y una relación amorosa producto de la cual nacen dos hijos. El vínculo se caracterizó por atribuciones de juicio de valor personal, a partir de la cuantía afectiva intercambiada por su ex pareja. Así, en la medida en que él se mostrara interesado en ella, ella se sentía valiosa como mujer y como persona. Simultáneamente, la familia extensa de la paciente juega un rol intrusivo y generan alianzas con las que se señala negativamente a la ex pareja de la doliente por causa de su trabajo como conductor, que le obliga a ausentarse con frecuencia de su núcleo. La paciente, por presión de su familia, aumenta los niveles de exigencia en dedicación al hogar para con su esposo, situación que deteriora el vínculo hasta romperlo por infidelidad de parte de él. Una vez se descubre el episodio, la doliente se muestra comprensiva e intenta recuperar el vínculo, pero su ex pareja se muestra reticente a intentarlo. Él se va de la casa y el hijo menor presenta problemas académicos y encopresis. La paciente establece una alianza con su hijo, con quien se identifica, de modo que suceden eventos como llorar juntos, orar porque el padre vuelva y, en general, ánimo depresivo compartido. En principio, asiste a consulta por las dificultades académicas del niño, pero por sugerencia terapéutica inicia su propio proceso. Muestra un ánimo depresivo la mayor parte del día, baja autoestima acentuada y catastrofización frente a la posibilidad de tener una calidad de vida satisfactoria en ausencia de su ex esposo.

Tabla No. 3 Intervención participante 3. No. de sesiones: 3.

\begin{tabular}{|l|l|}
\hline \multicolumn{1}{|c|}{ Actividad realizada } & \multicolumn{1}{|c|}{ Objetivo terapéutico } \\
\hline $\begin{array}{l}\text { Dibujo con la mano izquierda: técnica proyectiva que consiste en pedir al paciente que } \\
\text { dibuje un animal con su mano no dominante y cuente una historia asociada al dibujo. } \\
\text { La técnica explica que el contenido de dibujo y cuento se relacionan con el autocon- } \\
\text { cepto. Así, el terapeuta genera una lectura a manera de realimentación al paciente. }\end{array}$ & $\begin{array}{l}\text { Evaluar autoconcepto y } \\
\text { psicoeducar al paciente en la } \\
\text { relación que éste tiene con el } \\
\text { mantenimiento de su malestar. }\end{array}$ \\
\hline $\begin{array}{l}\text { Tarea terapéutica. Trabajo sobre auto-imagen: se le pide a la paciente que seleccione } \\
\text { con mayor detalle las prendas de uso diario, así como los accesorios y uso del cabe- } \\
\text { llo. Mirarse al espejo antes y después, de modo que obtenga Feedback positivo. }\end{array}$ & Modificación conductual. \\
\hline $\begin{array}{l}\text { Sesión de trabajo con su hijo menor. La paciente había establecido alianzas con } \\
\text { su hijo menor, quien se había identificado tanto con su dolor, que desarrolló un } \\
\text { cuadro de econpresis. Se fortalece un vínculo positivo, evidenciando que los inter- } \\
\text { cambios actuales se reducían a compartir expresiones de tristeza. }\end{array}$ & $\begin{array}{l}\text { Restituir el rol de madre sobre } \\
\text { su hijo. }\end{array}$ \\
\hline $\begin{array}{l}\text { Historizar: Se le pide a la paciente hablar con detalle de los éxitos conquistados } \\
\text { a lo largo de su historia de vida. Del mismo modo, se busca que la paciente com- } \\
\text { prenda que su ex esposo había tomado una decisión. }\end{array}$ & $\begin{array}{l}\text { Fortalecimiento de la } \\
\text { autoestima. }\end{array}$ \\
\hline $\begin{array}{l}\text { Balance decisional: La paciente no había tomado la decisión de asumir la pérdida. } \\
\text { Mediante la identificación de ganancias al mantener su conducta actual, sumado a la } \\
\text { evaluación de las probabilidades reales de reestablecimiento del vínculo, se le acompa- } \\
\text { ña a analizar la mejor opción entre continuar la relación o "dejar ir" a su ex esposo. }\end{array}$ & $\begin{array}{l}\text { Disminuir el nivel de malestar } \\
\text { por la ambivalencia actual de } \\
\text { esperar o no a su ex esposo. }\end{array}$ \\
\hline $\begin{array}{l}\text { Análisis de su estilo de vida. Mediante relatos de su cotidianidad la paciente } \\
\text { evidencia que tiene escasa red de apoyo. }\end{array}$ & $\begin{array}{l}\text { Evidenciar elicitadores de tristeza } \\
\text { y sensibilizar frente a la necesi- } \\
\text { dad de fortalecer red de apoyo. }\end{array}$ \\
\hline
\end{tabular}




\section{Participante 4}

Hombre de 31 años, profesional con Maestría.

Tipo de pérdida: Separación afectiva hace dos meses, con quien mantuvo una relación con expectativa ambivalente frente a la posibilidad de formalizar el vínculo durante ocho meses.

Religión: Agnóstico.

\section{Resumen de la Historia.}

El doliente refiere que la relación aparece en medio de un cese en sus entrenamientos cotidianos de natación, actividad frente a la cual expresa un muy alto nivel de satisfacción, al punto de hacer equivalencia con su felicidad. Se vincula poco a poco con su ex pareja en el contexto de paseos ocasionales en los que podía evidenciar el gusto que ella tenía por la natación y un estilo de vida que garantizaba un lugar secundario para la pareja. A propósito dice: "Me gustó de ella que no me necesitaba. Busco que esa persona tenga una vida. No me gusta que haga de mí su mundo. Me gusta el reto". Además del cumplimiento de tres requisitos que el doliente considera básicos para vincularse con una mujer como son: inteligencia, honestidad y nobleza.

La interpretación que hace de la relación al momento de iniciar el proceso se expresa en los siguientes términos: "Sentía que no era natural. Nos faltaba conocernos más. No quería que con ella pasara lo mismo que con otras personas (empezar mientras terminaba otra relación). Mi indicador de relación es dedicar tiempo y esto nos faltó un poco. Siento indiferencia aunque me da felicidad de que ella esté bien y yo también. Creo que lo que ella quería era experimentar".

La admiración por una pareja con vida independiente y autónoma trasciende a eventos como incomodarse cuando su ex novia dejaba prendas o artículos propios en su casa, frente a lo cual reaccionaba devolviéndoselos con premura.
Ella se encontraba terminando una relación de seis años, frente a la que evidenciaba agotamiento y desesperanza, situación que se convierte en la razón principal para no formalizar la relación con el doliente. A lo largo de la historia existen eventos en los que cesan los contactos, pero por iniciativa de ella se renuevan y la última vez con la promesa de concretar un vínculo de noviazgo. Transcurre una semana y ella decide ir de paseo con su ex-novio y otros amigos. Él intenta oponerse, no obstante, deja la decisión en manos de ella. Al regresar del paseo transcurre una semana sin contacto hasta que él envía un mensaje de texto en el que le hace ver que interpreta su silencio como una muestra de reinicio de la relación que ella guardaba con quien fuera su novio durante seis años. Ella devuelve el mensaje, dándole gracias por todo y reconociendo que él fue una muy buena experiencia.

Se refiere a ella en términos de ser única y la asemeja a su ex novia de siete años con quien mantuvo una relación de alta dedicación temporal, debido a que compartían espacios académicos y laborales. A propósito de la ex novia, con quien dura siete años, el doliente describe la relación en términos muy funcionales: "Ella me permitía sentirme como una mejor persona", además de referir alegría, comunicación, compartir gustos y acuerdo en metas laborales. La finalización de esta primera relación significativa llega con la enfermedad terminal de la mamá de ella y su posterior fallecimiento a los seis meses, situación que implicó dedicación casi exclusiva de la ex-novia y un distanciamiento de parte de él, con el propósito de evitar el dolor que significaba evidenciar el deterioro de su ex-suegra.

Ella hubiera podido ser Ana ${ }^{1}$ — se refiere a la mujer que genera el motivo de consulta-. Actualmente expresa gratitud por haberla conocido, pero sensación de desánimo y cansancio ante el hecho de pensarla constantemente. Con respecto a la posibilidad de volver a iniciar la relación, manifiesta poca esperanza: "Ella tendría que remar mucho y aunque nos encontráramos, creo que sucedería lo mismo".

1 Nombre ficticio que se le da a ex novia del doliente con quien mantuvo una relación de siete años. 


\section{Tabla No. 4 Intervención paciente 4 . No. de sesiones: 4}

\begin{tabular}{|l|l|}
\hline \multicolumn{1}{|c|}{ Actividad realizada } & \multicolumn{1}{|c|}{ Objetivo terapéutico } \\
\hline $\begin{array}{l}\text { Historizar: El paciente cuenta sus relaciones de pareja } \\
\text { más significativas y que generan activación emocional al } \\
\text { momento de compartir el relato. }\end{array}$ & $\begin{array}{l}\text { Facilitar la expresión emocional. Verificar en su historia } \\
\text { de aprendizaje elementos que impiden el mantenimiento } \\
\text { del vínculo. }\end{array}$ \\
\hline $\begin{array}{l}\text { Revisión estilo de vida actual: El paciente mantiene una } \\
\text { rutina de entrenamientos y deporte extremo que dificulta } \\
\text { el fortalecimiento de vínculo de pareja tradicional. }\end{array}$ & $\begin{array}{l}\text { Evidenciar que su estilo de vida contradice la propuesta } \\
\text { de pareja. }\end{array}$ \\
\hline Diálogo socrático sobre la responsabilidad de la ruptura. & $\begin{array}{l}\text { Reestructuración de la idea: "No sé qué fue lo que le } \\
\text { pasó a ella". }\end{array}$ \\
\hline
\end{tabular}

\section{Resultados}

1. Respecto al primer nivel de análisis, las respuestas cognitivas más frecuentes fueron: baja autoeficacia frente a la posibilidad de construir un nuevo vínculo afectivo, etiquetación negativa frente al rol cumplido hacia el otro, es decir, el doliente interpreta que lo que hizo o dejó de hacer durante la relación resultó en su totalidad insuficiente o inapropiado, razón por la cual, a su juicio, el vínculo se disolvió. También se encontró que presenta visión negativa de sí mismo (a), además de distraibilidad.

2. La respuesta emocional prevalente es el miedo al dolor, a no volver a encontrar una pareja, a no sobrevivir al duelo y la tristeza, aunque aparecen emociones complejas como la decepción y la culpa que asocian a pensamientos irracionales sobre las causas de la ruptura. Por ejemplo, pensar que se es absolutamente responsable o pensar que era responsabilidad de la otra persona, satisface todas las expectativas del doliente. Este tipo de respuestas afectivas suelen conservarse por mucho tiempo, complejizando la resolución del duelo.

3. A nivel comportamental se notó, predominantemente, nuevas formas de contacto que no son característicos ni de la pareja ni de la amistad, más bien, una relación ambigua en donde no existen conversaciones íntimas gratificantes, pero hay contacto físico sensual y sexual. Lo característico es que no pueden predecirse los encuentros, ni exigirse demandas afectivas. Esto obedece a un intento de no perder al otro, precisamente por evitar el malestar resultante de la ruptura. La relación con la red también resulta alterada. Así, el doliente se aísla o se acerca más de lo que lo hacía durante el vínculo. Se observaron comportamientos asociados a la búsqueda de espacios que fortalezcan la espiritualidad y la trascendencia, así como cambios en el uso del tiempo y el dinero.

4. A continuación se expondrán ideas centrales sobre la intervención, identificando la técnica específica que puede usarse para trabajar el objetivo terapéutico. Así:

- Se tiene muy presente una relación terapéutica que rescata la empatía. El doliente con frecuencia se queja de la imposibilidad de que su dolor sea comprendido en su contexto, de esta manera, el espacio terapéutico debe brindar la calidez suficiente como para que el paciente pueda hablar de su dolor.

- Identificación del objeto perdido y descongestión emocional: se trabaja a través 
de técnicas como la historización, que consiste en relatos que permitan conocer aspectos relevantes de la relación y de la persona ausente. Pueden darse instrucciones del tipo: "Cuenta todo lo que recuerdes de la persona". Los relatos pueden complementarse con fotos.

- Promoción de creencias saludables que aumenten la esperanza y fortalezcan el sentido de vida. Se trabajan mediante técnicas como guías deliberativas sobre esquemas de segundo orden, asociados a temas como vida, amor, muerte, divorcio, matrimonio, dignidad.

- Reestructuración sobre atribuciones disfuncionales de la pérdida, especialmente asociados a maximizaciones del valor del otro y el sentido de vida fundamentado en el otro.

- Reestructuración sobre atribuciones disfuncionales de la pérdida que generan culpa. Puede utilizarse técnica de evaluación de la relación, como la metáfora de la mesa, especialmente en divorcio o separación de pareja, o juegos como "el abogado defensor". ${ }^{2}$

- Desensibilización frente al sujeto perdido, ya que el doliente evita contextos asociados a su ex pareja. Puede trabajarse mediante la técnica de exposición a estímulos discriminativos, es importante hacerlo una vez haya pasado la congestión emocional.

- Fortalecimiento de red de apoyo mediante entrenamiento y ensayos conductuales en reclamos asertivos hacia su red de apoyo, cuando éstos tengan acciones que el doliente percibe como descuidos o incomprensiones de su dolor.

- Revisión del rol que actualmente se mantiene con su pareja. Se dialoga con el pa- ciente sobre la funcionalidad de la relación actual y el balance de satisfacción que trae el dar y el recibir, propuesto por el nuevo vínculo. El análisis muestra que es necesario señalar al doliente lo disfuncional de las ensoñaciones diurnas, es decir, las ilusiones de restaurar el vínculo.

- Fortalecimiento del amor propio. Puede utilizarse la técnica proyectiva del dibujo con la mano izquierda, mostrando al paciente aspectos de su autoconcepto que merecen ser trabajados. Vale aclarar que esta técnica se utiliza no con un objetivo diagnóstico, sino interventivo.

\section{Discusión y conclusiones}

El concepto de sí mismo se desestructura al momento de la ruptura, ya que la condición de estar en pareja determina rutinas, preferencias, elecciones, identidades aprendidas durante el vínculo. Una vez éste se disuelve, el doliente debe reorganizar dichas rutinas, gustos, identidades, etc. Por otro lado, la experiencia del dolor previo y posterior a la ruptura ubica al sujeto en contextos donde pueden aparecer respuestas nuevas que alteran lo que piensa de sí mismo. Este paso por situaciones de malestar y reorganización implican un alto costo afectivo, cognitivo y comportamental.

El proceso de elaboración del duelo resuelve tanto el malestar por la pérdida como el autoconcepto. Ahora bien, el malestar de la pérdida tiene un gradiente de normalidad, debido a que el dolor es natural. Lo que se busca, entonces, desde esta perspectiva, es reestructurar aquellos pensamientos que generan sufrimiento. Entendiendo por sufrimiento aquel malestar significativo que impide la elaboración de la pérdida y que lleva al sujeto o bien a la negación de la ruptura, a la desesperanza frente a la posibilidad de continuar la

2 Mesa: se realiza una metáfora de la relación de pareja como una mesa de cuatro patas compuestas por: afectividad, comunicación, sexualidad, economía. Ninguna de las patas es menos importante, las cuatro deben tener el tamaño suficiente para que la mesa funcione bien —el abogado defensor: el paciente debe asumir el rol de defender el argumento de "yo no tuve la culpa en... (aquello que el terapeuta identifica como irracional)—. (Piesschacon, 2005). 
vida e iniciar un nuevo vínculo, al resentimiento, al odio, a la victimización, desconfianza generalizada hacia el género o a la enfermedad física, entre otros.

Las explicaciones que el doliente da a la ruptura pueden generar sufrimiento, de modo que es necesario revisar los contenidos cognitivos que merecen ser trabajados para promover una saludable elaboración. La investigación actual muestra que el estilo cognitivo característico es de atribución externa, independiente de que el doliente sea quien toma o no la decisión de la ruptura. Este fenómeno se explica más desde el filtro de falacia de cambio (solo yo tuve la culpa/él es responsable de todo/dependo de él/ella para sentirme bien otra vez). De modo que es necesario que en el nivel terapéutico se trabajen contenidos asociados a la responsabilidad sobre la ruptura, el daño recibido, el valor de la otra persona (el ausente) en la vida personal y se hagan preguntas sobre proyecto de vida, sentido de vida, concepto del amor, de la pareja.
Cuando en el paciente existe dependencia afectiva, el proceso de elaboración tiende a complejizarse, de modo que el doliente puede mantenerse durante varios episodios "soportando" maltrato hasta que llega el día en el que "lo maltratan lo suficiente" y decide poner límite a su amor.

Cuando la ruptura aparece por infidelidad comprobada, resulta más evidente el rasgo de la negación de la pérdida y frecuente las conductas de búsqueda para solicitar o bien aclaración, o bien actos de reconquista que al resultar insuficientemente satisfactorias, o en el peor de los casos fallidas, motivaban la decisión de alejarse definitivamente. No obstante, esta intención cedía ante la nueva aparición de cualquier señal indicadora de probabilidad de regreso al vínculo.

El proceso de elaboración del duelo por ruptura de pareja no es exclusivo de quienes han sido "dejados", "abandonados" o "víctimas" de la decisión de su ex pareja, sino que también sucede con quienes deciden separase. 


\section{Referencias bibliográficas}

Boelen, P.A., Van den Bout, J., \& Van den Hout, M.A. (2006). Negative Cognitions and Avoidance in Emotional Problems after Bereavement: $A$ Prospective Study. Behavioural Reseach Therapy. (44) (11), 1657-72.

Boelen, P.A., De Keijser, J., Van den Hout, M.A., \& Van den Bout, J. (2011). Factors Associated with Outcome of Cognitive-Behaviorual Therapy for Complicated Grief: a Preliminar Study. Clinical Psychological Psychotherapy, (18) (4), 284-91.

Boelen, P.A., \& Van den Bout, J. (2002). Positive Thinking in Bereavement: Is It Related to Depression, Anxiety or Grief Symptomatology? Psychological Reports. (3), 857-63.

Cohen, J.A., Mannarino, A.P., \& Knudsen, K. (2004). Treating childhood traumatic grief: a pilot study. J Am Acad Child Adolesc Psychiatry. (43) (10),1225-33. Recuperado de http:// www.ncbi.nlm.nih.gov/pubmed/1538188 9 ? ordinalpos $=31$ \&itool $=$ EntrezSystem 2 . PEntrez.Pubmed.Pubmed_ResultsPanel. Pubmed_RVDocSum

Cozolino, L. (2003). Los psicoterapeutas redescubren el cerebro. Project syndicate. A world of ideas. Recuperado de http:/ / www.project-syndicate.org/commentary/cozolino1/Spanish

Crenshaw, D. (2006). An interpersonal neurobiological-informed treatment model for childhood traumatic grief. Omega (Westport). (54)(4), 319-35. Recuperado de www.ncbi.nlm.nih.gov/pu $\mathrm{bmed} / 18186426$ ? ordinalpos $=2 \&$ itool $=\mathrm{E}$ ntrezSystem2.PEntrez.Pubmed.Pubmed_ ResultsPanel.Pubmed_RVDocSum

Gotta, G., Green, R.J., Rohblum, E., Solomon, S., Balsam, K., \& Schaertz, P. (2011).
Heterosexual, lesbian, and Gay Male Relationships: A Comparison of Couples in 1975 and 2000. Family Process. (50) (3), 353-76.

Grupo de Investigadores en psicología. (2008). Disertaciones académicas. Bogotá: Universidad Manuela Beltrán.

Heiney, S.P., Dunaway, N.C., \& Webster, J. (1995). Good grieving-an intervention program for grieving children. Oncol Nurs Forum, (22) (4), 64955. Recuperado de http://www.ncbi.nlm.nih. gov/pubmed/7675667

Kranzler, E.M., Shaffer, D., Wasserman, G., \& Davies, M. (1990). Early childhood bereavement J Am Acad Child Adolesc Psychiatry, (29)(4), 51320. Recuperado de http://www.ncbi.nlm.nih. gov/pubmed/2387784

Love, A. (2007). Progress in understanding grief, complicated grief, and caring for the bereaved Contemp Nurse, 27(1), 73-83. Recuperado de http:// www.ncbi.nlm.nih.gov/pubmed/183869 57 ?ordinalpos $=2 \&$ itool $=$ EntrezSystem 2 . PEntrez.Pubmed.Pubmed_ResultsPanel. Pubmed_RVDocSum

Martínez, P. (2006). El método de estudio de caso. Estrategia metodológica de la investigación científica. Pensamiento y Gestión, 20, 165193.

Piesschacon, M. (2005). Apuntes de Clase. Documento no publicado. Maestría en Psicología Clínica Universidad Católica de Colombia. Bogotá.

Riso, W. (2006). Manual de Terapia cognitiva. Bogotá: Norma. 